\title{
Condições de temperatura, umidade relativa e atmosfera controlada para o armazenamento de cebolas da cultivar 'Crioula'
}

\author{
Temperature, relative humidity and controlled atmosphere conditions to storage 'Crioula' onions
}

\author{
Auri Brackmann ${ }^{\mathrm{I} *}$ Adriano Roque de Gasperin ${ }^{\mathrm{I}}$ Anderson Weber ${ }^{\mathrm{I}}$ \\ Rogério de Oliveira Anese ${ }^{I}$
}

\section{RESUMO}

O objetivo deste trabalho foi de avaliar condições de armazenamento para ampliar o período de pós-colheita de cebola da cultivar 'Crioula'. Para tanto, foram executados três experimentos para avaliar o efeito da temperatura, umidade relativa (UR) e atmosfera controlada (AC): experimento 1 (diferentes temperaturas): [1] $-0,5^{\circ} \mathrm{C},[2] 0,5^{\circ} \mathrm{C}$, [3] $2^{\circ} \mathrm{C}$, [4] $4^{\circ} \mathrm{C}$, [5] $6^{\circ} \mathrm{C}$ e [6] $10^{\circ} \mathrm{C}$; experimento 2 (níveis de UR): [1] $70 \%$, [2] 80\% e [3] 90\%; e experimento 3 (condições de AC): [1] $21 \mathrm{kPa} \mathrm{O}+0,03 \mathrm{kPaCO}$, [2] $0,5 \mathrm{kPa} \mathrm{O}+0 \mathrm{kPa} \mathrm{CO}$, [3] $1,0 \mathrm{kPaO}+0 \mathrm{kPaCO}$, [4] 2,0kPa $\mathrm{O}_{2}+\mathrm{OkPa} \mathrm{CO}_{2}$, [5] 1,0kPa $\mathrm{O}_{2}+2,0 \mathrm{kPaCO} \mathrm{CO}_{2}$ e [6]1,0kPa O$+4,0 \mathrm{kPa} \mathrm{CO}_{2}$. O delineamento experimental utilizado foi o inteiramente casualizado. Após seis meses de armazenamento, foram realizadas as análises no momento da saída dos bulbos das câmaras e após 15 dias de exposição a $20^{\circ} \mathrm{C}$. A brotação e a podridão foram inibidas na temperatura de $0,5^{\circ} \mathrm{C}$, diferentemente das temperaturas iguais e superiores a $4^{\circ} \mathrm{C}$, em que mais de $90 \%$ dos bulbos brotaram. As UR de 70 e $80 \%$ foram melhores, pois ocorreu menor brotação. O baixo oxigênio controlou a brotação dos bulbos, proporcionando maior número de bulbos comerciáveis após seis meses em AC e também após 15 dias de exposição a $20^{\circ} \mathrm{C}$.

Palavras-chave: Allium cepa L, temperatura, umidade relativa, atmosfera controlada.

\section{ABSTRACT}

The aim of this research was to evaluate conditions to maintain postharvest quality of 'Crioula' onions. Three experiments were done, evaluating the effect of temperature, relative humidity $(\mathrm{RH})$ and controlled atmosphere (CA): different temperatures: [1] $-0.5^{\circ} \mathrm{C}$, [2] $0.5^{\circ} \mathrm{C},[3] 2^{\circ} \mathrm{C},[4] 4^{\circ} \mathrm{C}$, [5] $6^{\circ} \mathrm{C}$ and [6] $10^{\circ} \mathrm{C}$. Levels of $\mathrm{RH}$ : [1] $70 \%$, [2] $80 \%$ and [3] $90 \%$; and different CA conditions: [1] $21 \mathrm{kPa} \mathrm{O}+0.03 \mathrm{kPa} \mathrm{CO}$, [2]
$0.5 \mathrm{kPaO} \mathrm{O}_{2}+0 \mathrm{kPaCO}$, [3] $1.0 \mathrm{kPaO}+0 \mathrm{kPaCO}$, [4] $2.0 \mathrm{kPa}$ $\mathrm{O}_{2}+0 \mathrm{kPaCO}$, [5] $1.0 \mathrm{kPa} \mathrm{O}_{2}+2.0 \mathrm{kPaCO} \mathrm{CO}_{2}$ and [6] $\mathrm{O}_{2}$ $1.0 \mathrm{kPa}+4.0 \mathrm{kPa} \mathrm{CO}_{2}$. The experimental design was completely randomized. Ripening and quality evaluations were carried out after six months of storage more fifteen days at $20^{\circ} \mathrm{C}$. The sprout and rot were inhibited at a temperature of $0.5^{\circ} \mathrm{C}$, unlike the equal and temperatures above $4^{\circ} \mathrm{C}$, in which more than $90 \%$ of the bulbs have sprouted. The RH of 70 and $80 \%$ were better, because there was less shooting. The low oxygen controlled the sprouting of bulbs, providing a major number of marketable bulbs after six months in CA and also after 15 days at $20^{\circ} \mathrm{C}$.

Key words: Allium cepa L, temperature, relative humidity, controlled atmosphere.

\section{INTRODUÇÃO}

No panorama mundial, a produção de cebola (Allium cepa L) aumentou cerca de $25 \%$ na última década, sendo a segunda hortaliça em importância econômica (BOITEUX \& MELO, 2004). No Brasil, embora a produção de cebolas concentra-se em um pequeno número de Estados produtores, nos últimos anos, a cultura da cebola ampliou-se em área plantada de forma significativa. No entanto, por ser um produto altamente perecível e com nível de demanda em descompasso com o crescimento da oferta, no cultivo da cebola, ocorrem muitas perdas pós-colheita, provocadas principalmente pela sua inadequada condição de armazenamento (CAMARGO, 2003). O armazenamento figura como uma importante ferramenta

IDepartamento de Fitotecnia, Centro de Ciências Rurais (CCR), Universidade Federal de Santa Maria (UFSM). Av. Roraima, ${ }^{\circ}$ 1000, Cidade Universitária, Bairro Camobi, 97105-900, Santa Maria, RS, Brasil. E-mail: auribrackmann@gmail.com. *Autor para correspondência. 
dos produtores, pois possibilita o aumento da vida pós-colheita do bulbo, permitindo assim a oferta de cebolas de qualidade ao mercado consumidor na entressafra e equalizando os períodos de excesso e carência de oferta (MORETTI, 2004). Entretanto, há poucas informações na literatura sobre as melhores condições de temperatura, umidade relativa (UR) do ar e condições de atmosfera controlada (AC) para o armazenamento de cebola da cultivar 'Crioula'.

Durante o armazenamento, as baixas temperaturas retardam os processos fisiológicos e bioquímicos de amadurecimento dos frutos e vegetais, principalmente a respiração (KADER, 1986). Para bulbos e tubérculos, as baixas temperaturas têm importante efeito sobre a dormência e o crescimento por brotamento, já que todas as atividades metabólicas podem ser reduzidas pelo efeito das baixas temperaturas (CHITARRA \& CHITARRA, 2005).

A brotação é um dos principais fatores limitantes na conservação da cebola após a colheita. O armazenamento refrigerado, mesmo em temperaturas próximas a $0^{\circ} \mathrm{C}$, induz a quebra de dormência e facilita a brotação, quando o bulbo volta a ser exposto a temperaturas próximas a $20^{\circ} \mathrm{C}$ (LUENGO \& CALDO, 2001). Uma maneira de diminuir os danos provocados pela baixa temperatura e evitar o desenvolvimento de microrganismos, brotamento e murchamento é o controle da umidade relativa do ar. A UR recomendada para a maioria dos produtos perecíveis em câmaras de armazenamento é de 90 a 95\%, porém há exceções, como alho e cebola, que devem ser armazenados em UR mais baixa (KADER, 2002).

Em suplemento à refrigeração prolongada, a atmosfera controlada pode ser usada na conservação tanto no armazenamento, como durante o transporte de diversos produtos hortícolas (LANA \& FINGER, 2000). A atmosfera controlada consiste em reduzir a concentração de $\mathrm{O}_{2}$ e aumentar a de $\mathrm{CO}_{2}$, visando a retardar a senescência, por meio da redução do processo respiratório, já que as trocas gasosas decorrente da respiração são diminuídas, além do baixo teor de $\mathrm{O}_{2}$ inibir a cadeia respiratória (BRACKMANN \& CHITARRA, 1998). Esses mesmos autores afirmam que os baixos níveis de $\mathrm{O}_{2}$ e altos de $\mathrm{CO}_{2}$ reduzem a síntese e a ação do etileno sobre o metabolismo de fruto e vegetais e diminuem a ocorrência de podridões.

O objetivo deste trabalho foi determinar as condições de temperatura, umidade relativa e atmosfera controlada para o armazenamento de cebolas da cultivar 'Crioula'.

\section{MATERIAL E MÉTODOS}

O experimento foi conduzido no Núcleo de Pesquisa em Pós-colheita (NPP) do Departamento de
Fitotecnia da Universidade Federal de Santa Maria (UFSM), no ano de 2006. O material experimental constou de bulbos de cebola da cultivar 'Crioula', oriundos de plantações comerciais da Ilha dos Marinheiros, em Rio Grande, no Rio Grande do Sul (RS).

O trabalho foi dividido em três experimentos, em que foram avaliados, em experimentos distintos, os seguintes parâmetros: temperaturas, níveis de umidade relativa (UR) e atmosfera controlada (AC). Nos três experimentos, os bulbos foram armazenados em minicâmaras experimentais com volume de $0,185 \mathrm{~m}^{-3}$, e os bulbos do experimento em AC tiveram as minicâmaras hermeticamente vedadas.

No experimento 1, com diferentes temperaturas, as condições avaliadas foram: [1] $-0,5^{\circ} \mathrm{C}$, [2] $0,5^{\circ} \mathrm{C}$, [3] $2^{\circ} \mathrm{C}$, [4] $4^{\circ} \mathrm{C}$, [5] $6^{\circ} \mathrm{C}$ e [6] $10^{\circ} \mathrm{C}$. As temperaturas das câmaras frigoríficas foram reguladas automaticamente por meio de termostatos de alta precisão e acompanhadas diariamente por meio de termômetros com bulbo de mercúrio inseridos na polpa de alguns bulbos.

Para o experimento 2, com níveis de umidade relativa, foram avaliadas as seguintes condições: [1] $70 \%$, [2] $80 \%$ e [3] 90\%, em que a temperatura de armazenamento foi de $0,5^{\circ} \mathrm{C}$. Para manter baixa a umidade relativa durante o armazenamento dos bulbos, foi utilizada uma bomba de membrana, que circulava o ar do interior da minicâmara para dentro de um recipiente contendo sílica gel, a qual era substituída após sua saturação. Além disso, instalou-se um psicrômetro no interior das minicâmara, para acompanhar a UR.

No experimento 3, com condições de AC, os tratamentos avaliados foram: [1] $21 \mathrm{kPa} \mathrm{O}_{2}+0,03 \mathrm{kPa} \mathrm{CO}_{2}$, [2] 0,5 $\mathrm{kPa} \mathrm{O}_{2}+0 \mathrm{kPa} \mathrm{CO}_{2}$, [3] $1,0 \mathrm{kPa} \mathrm{O}_{2}+0 \mathrm{kPa} \mathrm{CO}_{2}$, [4] $2,0 \mathrm{kPa} \mathrm{O}_{2}+0 \mathrm{kPa} \mathrm{CO}_{2}$, [5] 1,0kPa O$+2,0 \mathrm{kPa} \mathrm{CO}_{2} \mathrm{e}$ [6] $1,0 \mathrm{kPa} \mathrm{O}_{2}+4,0 \mathrm{kPa} \mathrm{CO}_{2}$. A temperatura e a UR foram de $0,5^{\circ} \mathrm{C}$ e $95 \%$, respectivamente. As condições de atmosfera controlada foram estabelecidas um dia após a instalação do experimento, mediante diluição do $\mathrm{O}_{2}$ por meio da injeção de nitrogênio no interior das minicâmaras, o qual foi obtido por um gerador que utiliza o princípio de peneira molecular (Pressure Swing Adsorption, PSA), e posterior injeção de $\mathrm{CO}_{2}$, proveniente de cilindros de alta pressão, até atingir os níveis estabelecidos nos tratamentos. Essas condições foram monitoradas e corrigidas diariamente por meio de analisadores eletrônicos de fluxo contínuo da marca Agri-datalog. O oxigênio, consumido pela respiração dos bulbos, foi compensado por meio da injeção de ar nas câmaras. O dióxido de carbono em excesso, resultante do processo respiratório, foi absorvido por uma solução de hidróxido de sódio, pela qual foi circulado o ar das câmaras. 
Condições de temperatura, umidade relativa e atmosfera controlada para o armazenamento de cebolas...

Após seis meses, foram realizadas as análises laboratoriais no momento da saída dos bulbos das câmaras e após 15 dias de exposição à temperatura de $20^{\circ} \mathrm{C}$, exceto para o experimento 1 , que foi avaliado somente na saída da câmara. Os parâmetros avaliados foram brotação: os bulbos que apresentavam brotamento externo foram contabilizados, e os resultados foram apresentados em termos de porcentagem de bulbos brotados; podridões: determinada pela contagem dos bulbos que apresentavam lesões com diâmetro superior a $5 \mathrm{~mm}$ e com sintomas característicos de ataques de fungos, sendo expressa em porcentagem de bulbos podres; comerciáveis: bulbos com ausência de brotamento e podridão; firmeza do bulbo: avaliada na região equatorial dos bulbos, em lados opostos, com um penetrômetro manual com ponteira de $11,5 \mathrm{~mm}$ de diâmetro e os resultados expressos em Newton (N); sólidos solúveis (SS): determinados com um refratômetro manual com correção da temperatura e expressos em ${ }^{\circ}$ Brix; acidez titulável: determinada pela titulação de uma solução contendo $10 \mathrm{~mL}$ de suco diluído em $100 \mathrm{~mL}$ de água destilada com $\mathrm{NaOH}(0,1 \mathrm{~N})$ até $\mathrm{pH}$ 8,1, sendo os resultados expressos em meq $100 \mathrm{~mL}^{-1}$; respiração: determinada pela produção de $\mathrm{CO}_{2}$ por meio da leitura do ar do espaço livre de uma amostra de cerca de $1000 \mathrm{~g}$ de frutos, fechados hermeticamente em recipientes de cinco litros por aproximadamente quatro horas a $20^{\circ} \mathrm{C}$. A atmosfera do recipiente foi circulada por meio de um analisador eletrônico de $\mathrm{CO}_{2}$, marca Agri-Datalog, sendo os dados expressos em $\mathrm{mL}$ $\mathrm{CO}_{2} \mathrm{~kg}^{-1} \mathrm{~h}^{-1}$.

O delineamento experimental utilizado foi o inteiramente casualizado, com quatro repetições para os experimentos 1 e 3 , e sete repetições para o experimento 2, sendo as unidades experimentais compostas por 20 bulbos. Os dados expressos em porcentagem foram transformados pela fórmula arc.sen $\sqrt{x / 100}$, antes de realizar a análise da variância. As médias foram comparadas entre si pelo teste de Tukey, em nível de 5\% de probabilidade de erro.

\section{RESULTADOS E DISCUSSÃO}

O experimento com diferentes temperaturas mostrou que, na temperatura de $0,5^{\circ} \mathrm{C}, 100 \%$ dos bulbos apresentaram condições de comercialização apresentando diferença significativa com relação à temperatura de $-0,5^{\circ} \mathrm{C}$ e às temperaturas superiores a $0,5^{\circ} \mathrm{C}$ (Tabela 1). LUENGO \& CALDO (2001) afirmam que a cebola deve ser armazenada em temperaturas superiores ao ponto de congelamento $\left(-0,8^{\circ} \mathrm{C}\right)$ e ao redor de $0^{\circ} \mathrm{C}$, em que a brotação fica estacionada, concordando com os resultados deste trabalho, em que, na temperatura de $0,5^{\circ} \mathrm{C}$, não ocorreu brotação dos bulbos, provavelmente devido à baixa atividade metabólica, uma vez que o material vegetal apresentou baixo índice respiratório e não ocorreu podridão nos bulbos. Já a brotação dos bulbos foi maior na temperatura de $10^{\circ} \mathrm{C}$, não diferindo das temperaturas de 6 e $4^{\circ} \mathrm{C}$ (Tabela 1). Segundo MIEDEMA (1994), temperaturas entre 5 e $15^{\circ} \mathrm{C}$ aceleram a brotação e, por isso, devem ser evitadas durante o armazenamento.

No experimento com diferentes níveis de UR do ar na saída da câmara e aos 15 dias a $20^{\circ} \mathrm{C}$, observouse que tanto 70 , como $80 \%$ de UR não apresentam diferença estatística significativa entre si, em relação a todos os parâmetros analisados (Tabela 2). Segundo LUENGO \& CALDO (2001), a cebola deve ser armazenada sob umidade relativa ao redor de $70 \%$; entretanto, a vida útil da cebola armazenada depende muito da cultivar. As cultivares que melhor se conservam são aquelas com alto teor de matéria seca (THOMPSON et al., 1972). Para a UR de 70 e 80\%, os dados mostram que, na saída da câmara, a firmeza teve média menor e estatisticamente diferente em comparação com $90 \%$ de UR; entretanto, após o período de exposição a $20^{\circ} \mathrm{C}$, a firmeza apresentou-se maior em condições de baixa umidade relativa. A firmeza do bulbo pode ter sido influenciada pela perda de água para a atmosfera externa, em razão dos diferentes níveis de umidade.

Tabela 1 - Qualidade de cebola da cultivar ‘Crioula’, após seis meses de armazenamento em diferentes temperaturas. Santa Maria, RS, 2009.

\begin{tabular}{|c|c|c|c|c|}
\hline Temperatura & Brotação (\%) & Podridões (\%) & Comerciáveis (\%) & Respiração $\left(\mathrm{mLCO}_{2} \mathrm{~kg}^{-1} \mathrm{~h}^{-1}\right)$ \\
\hline$-0,5$ & $33,8 c^{*}$ & $0,00 \mathrm{c}$ & $66,3 \mathrm{~b}$ & 10,9 bc \\
\hline 0,5 & $0,00 \mathrm{~d}$ & $0,00 \mathrm{c}$ & $100,0 \mathrm{a}$ & $7,57 \mathrm{c}$ \\
\hline 2 & $67,5 \mathrm{~b}$ & $0,00 \mathrm{c}$ & 32,5 c & $23,0 \mathrm{ab}$ \\
\hline 4 & 91,3 a & 36,3 a & $8,75 \mathrm{~d}$ & 18,9 abc \\
\hline 6 & 90,0 a & $15,0 \mathrm{~b}$ & $10,0 \mathrm{~d}$ & $26,4 \mathrm{a}$ \\
\hline 10 & 95,0 a & $10,0 \mathrm{~b}$ & 0,00 e & $27,1 \mathrm{a}$ \\
\hline CV (\%) & 14,4 & 28,7 & 15,6 & 33,9 \\
\hline
\end{tabular}

*Tratamentos com médias não seguidas pelas mesmas letras, na vertical, diferem estatisticamente pelo teste Tukey, a 5\%. 
Tabela 2 - Qualidade de cebola da cultivar 'Crioula' após seis meses de armazenamento, em três níveis de umidade relativa na saída da câmara e aos 15 dias, a 20C. Santa Maria, RS, 2009.

\begin{tabular}{|c|c|c|c|c|c|c|}
\hline $\begin{array}{l}\text { UR } \\
\text { (\%) }\end{array}$ & $\begin{array}{l}\text { Firmeza } \\
(\mathrm{N})\end{array}$ & $\begin{array}{c}\text { Brotação } \\
(\%)\end{array}$ & $\begin{array}{l}\text { Podridões } \\
\text { (\%) }\end{array}$ & $\begin{array}{c}\text { Comerciáveis } \\
\text { (\%) }\end{array}$ & $\begin{array}{c}\text { SST } \\
\left({ }^{\circ} \text { Brix }\right)\end{array}$ & $\begin{array}{l}\text { Acidez titulável } \\
\left.\text { (meq100 } \mathrm{mL}^{-1}\right)\end{array}$ \\
\hline 70 & $76,7 \mathrm{~b}^{*}$ & $0,00 \mathrm{~b}$ & $1,25 \mathrm{a}$ & 98,8 a & $10,4 \mathrm{a}$ & 2,30 a \\
\hline 80 & $79,8 \mathrm{~b}$ & $0,00 \mathrm{~b}$ & 3,75 a & 96,3 a & 9,95 a & $2,29 \mathrm{a}$ \\
\hline 90 & 89,2 a & 66,3 a & 3,75 a & $33,8 \mathrm{~b}$ & $10,0 \mathrm{a}$ & $2,10 \mathrm{~b}$ \\
\hline CV (\%) & 4,87 & 25,9 & 51,0 & 10,5 & 3,34 & 3,53 \\
\hline 70 & $74,1 \mathrm{ab}$ & $12,5 \mathrm{~b}$ & 7,50 a & 80,0 a & 10,9 a & $2,40 a b$ \\
\hline 80 & 77,5 a & $12,5 \mathrm{~b}$ & 6,25 a & 81,2 a & $10,7 \mathrm{a}$ & $2,61 \mathrm{a}$ \\
\hline 90 & $70,1 \mathrm{~b}$ & $71,2 \mathrm{a}$ & 3,75 a & $25,0 \mathrm{~b}$ & $10,8 \mathrm{a}$ & $2,22 \mathrm{~b}$ \\
\hline CV (\%) & 4,52 & 19,4 & 50,3 & 14,2 & 3,23 & 7,49 \\
\hline
\end{tabular}

* Tratamentos com médias não seguidas pelas mesmas letras, na vertical, diferem estatisticamente pelo teste Tukey, a 5\%.

O percentual de bulbos brotados foi menor com a utilização de baixa UR, tanto na saída da câmara, onde não houve brotação, quanto após 15 dias a $20^{\circ} \mathrm{C}$ (Tabela 2). Esse resultado está em acordo com o reportado por LIMA (2009), que recomenda o uso da UR em razão da menor ocorrência de brotação. Isso pode ser explicado pelo fato de que a maior quantidade de vapor d'água presente e disponível no ar resultou em uma melhor condição para a atuação de enzimas e hormônios, causando o crescimento de brotos. Com isso, as condições de 70 e $80 \%$ de UR proporcionaram maior percentual de bulbos em condições de serem comercializados (Tabela 2). Para TANAKA (1991), a umidade relativa elevada promove um maior efeito decisivo na brotação dos bulbos do que o fator temperatura, além de facilitar o ataque de agentes causadores de podridões. A acidez titulável foi maior nos bulbos que ficaram submetidos à menor UR. Isso se deve possivelmente pela concentração de ácidos, uma vez que, quanto menor a UR do ar, menor o conteúdo de vapor d'água do ar, menor a saturação do ar e assim há um maior número de partículas de água que evaporam do produto (bulbos), em comparação com uma condição de UR maior. O teor de SST e a incidência de podridões não diferiram estatisticamente.

No experimento em AC, o baixo oxigênio (1,0kPa ou menos) proporcionou maior número de bulbos sadios (comerciáveis), apresentando um efeito significativo desses tratamentos se comparados com as concentrações de 2,0 e $21 \mathrm{kPa}$ de $\mathrm{O}_{2}$ Os tratamentos com $1 \mathrm{kPa}$ de $\mathrm{O}_{2}$ também foram estatisticamente eficientes no controle da brotação (Tabela 3). Níveis de $1 \mathrm{kPa}$ de oxigênio inibem a respiração e a germinação em comparação com $21 \mathrm{kPa}$ de oxigênio (PRAEGER et al., 2003). Isso se deve ao baixo nível de oxigênio na câmara que reduz a respiração celular, diminuindo o fornecimento de energia necessário para que ocorra a divisão celular e consequentemente o crescimento de brotos. Segundo SUMNER (2000), o armazenamento em AC pode limitar o período de vida útil, acelerando a brotação três semanas após a remoção da condição de AC. No entanto, alguns autores alegam que a AC prolonga a vida de prateleira das cebolas durante três semanas a $18^{\circ} \mathrm{C}$, após 36 semanas de armazenamento em AC com $1 \mathrm{kPa}$ ou $0,5 \mathrm{kPa}$ de $\mathrm{O}_{2}$ mais $\mathrm{CO}_{2}<0,3 \mathrm{kPa}$, se comparadas com as armazenadas em ar (PRAEGER et al., 2003). O presente trabalho mostra que, após 15 dias de exposição a $20^{\circ} \mathrm{C}$ dos bulbos, a AC não acelerou a brotação dos bulbos, como relata SUMNER (2000), e sim manteve um alto número de bulbos sadios, concordando com PRAEGER et al. (2003). Com relação aos níveis de $\mathrm{CO}_{2}$ testados, não houve influência em nenhum parâmetro.

\section{CONCLUSÃO}

O armazenamento prolongado da cebola da cultivar 'Crioula' deve ser realizado sob temperatura de $0,5^{\circ} \mathrm{C}$, em que a brotação fica paralisada e ocorre menor incidência de podridões. A UR deve ser mantida entre 70 ou até $80 \%$, proporcionando bulbos mais sadios e de melhor qualidade. A AC complementa o armazenamento refrigerado, prolongando a vida de prateleira da cebola, além de, com $1 \mathrm{kPa}$ de $\mathrm{O}_{2}$ ou menos, retardar a brotação em temperatura ambiente após a saída da câmara. 
Tabela 3 - Qualidade de cebola da cultivar 'Crioula' após seis meses de armazenamento, em diferentes condições de atmosfera controlada, saída da câmara e aos 15 dias, a $20^{\circ} \mathrm{C}$. Santa Maria, RS, 2009.

\begin{tabular}{|c|c|c|c|c|}
\hline \multicolumn{2}{|c|}{----------Condições de AC---------- } & \multirow{2}{*}{$\begin{array}{c}\text { Brotação } \\
(\%)\end{array}$} & \multirow{2}{*}{$\begin{array}{l}\text { Podridões } \\
\text { (\%) }\end{array}$} & \multirow{2}{*}{$\begin{array}{c}\text { Comerciáveis } \\
\text { (\%) }\end{array}$} \\
\hline $\mathrm{kPa} \mathrm{O}_{2}$ & $\mathrm{kPa} \mathrm{CO}_{2}$ & & & \\
\hline & & \multicolumn{3}{|c|}{----------------------------------- Saída da câmara ---------------------------------- } \\
\hline 21 & 0,03 & $93,8 a^{*}$ & $1,25 \mathrm{ab}$ & $5,00 \mathrm{~b}$ \\
\hline 0,5 & 0,0 & $1,25 \mathrm{c}$ & 6,25 a & 93,8 a \\
\hline 1,0 & 0,0 & $31,3 \mathrm{~b}$ & $0,00 \mathrm{~b}$ & 68,8 a \\
\hline 2,0 & 0,0 & 77,5 a & $5,00 \mathrm{a}$ & $21,3 \mathrm{~b}$ \\
\hline 1,0 & 2,0 & $5,00 \mathrm{c}$ & $1,25 \mathrm{ab}$ & 92,5 a \\
\hline 1,0 & 4,0 & 7,50 bc & $2,50 \mathrm{ab}$ & 90,0 a \\
\hline \multicolumn{2}{|c|}{ CV (\%) } & 31,2 & 60,6 & 18,3 \\
\hline & & \multicolumn{3}{|c|}{ 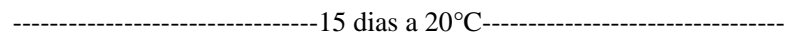 } \\
\hline 21 & 0,03 & 93,8 a & $1,25 \mathrm{~b}$ & $5,00 \mathrm{~b}$ \\
\hline 0,5 & 0,0 & $6,25 \mathrm{~b}$ & 17,5 a & 76,3 a \\
\hline 1,0 & 0,0 & $31,3 \mathrm{~b}$ & $3,75 \mathrm{ab}$ & 65,0 a \\
\hline 2,0 & 0,0 & 80,0 a & $3,75 a b$ & $16,3 \mathrm{~b}$ \\
\hline 1,0 & 2,0 & $7,50 \mathrm{~b}$ & $5,00 \mathrm{ab}$ & 87,5 a \\
\hline 1,0 & 4,0 & $7,50 \mathrm{~b}$ & $10,0 \mathrm{ab}$ & 82,5 a \\
\hline \multicolumn{2}{|c|}{ CV (\%) } & 32,5 & 61,5 & 23,7 \\
\hline
\end{tabular}

* Tratamentos com médias não seguidas pelas mesmas letras, na vertical, diferem estatisticamente pelo teste Tukey, a 5\%.

\section{REFERÊNCIAS}

BOITEUX, L.S.; MELO, P.C.T. Sistema de produção de cebola (Allium cepa L): taxonomia e origem. 2004. v.5. Disponível em: <http://www.cnph.embrapa.br/sistprod/cebola/ index.htm>. Acesso em: 21 set. 2009.

BRACKMANN, A.; CHITARRA, A.B. Atmosfera controlada e atmosfera modificada. In: BORÉM, F.M. (Coord.). Armazenamento e processamento de produtos agrícolas. Lavras: UFLA/SBEA, 1998. p.133-169.

CAMARGO, J.P. A produção de cebola no Brasil. Revista Batata Show, Ano 3, n.7, 41p, 2003. Disponível em: <http:/ /www.abbabatatabrasileira.com.br/revista07_023.htm>. Acesso em: 21 set. 2009

CHITARRA, M.I.F.; CHITARRA, A.B. Pós-colheita de frutas e hortaliças: fisiologia e manuseio. Lavras: UFLA, 2005. 783p.

KADER, A.A. Biochemical and physiological basis for effects of controlled and modified atmospheres on fruits and vegetables. Food technology, v.40, n.5, p.99-104, 1986.

KADER, A.A. Postharvest technology of horticultural crops. 3.ed. Califórnia: Division of Agriculture and Natural Resources, 2002. 535p.

LANA, M.M.; FINGER, F.L. Atmosfera modificada e controlada: aplicação na conservação de produtos hortícolas. Brasília: Embrapa Comunicação para Transferência de Tecnologia/ Embrapa Hortaliças, 2000. 34p.
LIMA, M.A.C. Colheita e manuseio pós-colheita. Cultivo de cebola no Nordeste. Disponível em: <http:// sistemasdeprodcao.cnptia.embrapa.br/FontesHTML/Cebola/ CultivoCebolaNordeste/colheita.htm>. Acesso em: 5 ago. 2009.

LUEnGO, R. de F.A.; CALDO, A.G. Armazenamento de hortaliças. Brasília, DF: Embrapa Hortaliças, 2001. 242p.

MIEDEMA, P. Bulb dormancy in onion. I. The effects of temperature and cultivar on sprouting and rooting. Journal of Horticultural Science, v.69, n.1, p.29-39, 1994.

MORETTI, C.L. Colheita e manuseio pós-colheita. Sistema de produção de cebola (Allium cepa L), 2004. Disponível em: <http://www.cnph.embrapa.br/sistprod/cebola/ colheita_e_pos.htm>. Acesso em: 21 set. 2009.

PRAEGER, U. et al. Effects of ultra low oxygen storage on postharvest quality of onion bulbs (Allium cepa L. var. cepa). European Journal of Horticultural Science, v.68 p.1419, 2003.

SUMNER, P.E. Vidalia onion CA storage requirements. Georgia: College of Agricultural and Environmental Sciences, University of Georgia, 2000. Disponível em: <http://www.tifton.uga.edu/sumner/ tifton/Veg/CAOnionREQ.htm>. Acesso em: 27 ago. 2009.

TANAKA, M. Studies on the storage of onion bulbs harvested in autumn. Hokkaido National Agricultural Experiment Station, n.156, p.119-122, 1991.

THOMPSON, A.K. et al. Onion storage in the tropics. Tropical Science, v.14, n.1, p.19-34, 1972. 